\title{
Development of Mathematical Model to Control Human Population
}

\author{
Rakesh Yadav
}

School of Chemical Engineering \& Physical Sciences, Lovely Professional University, Punjab, India

Corresponding author: rakesh.21798@lpu.co.in

\begin{abstract}
In this article an effort has been made for a stochastic model of intrinsic rate of human population growth of India and studied when the couples at the personal level decide whether to continue child bearing or not is made on the basis of desired number of male Childs. The aim of developing such a model of intrinsic rate of population growth in terms of bio-social parameters is to study the growth rate of ultimate stable population implied when couples adopt family building process based on desired number of living male child. The departure of the proposed formulation from traditional approach makes it more realistic and relevant from the point of view of bio-social parameters shaping human reproduction. In terms of long run nature of growth rate of population. This model explains the sensitiveness of the biosocial parameters such as female's age at marriage time, survival chance of mother, spacing between births etc. It will also help to stimulate the consequences of alternating bio-social parameters shaping human reproduction
\end{abstract}

Keywords: Human reproduction, Early childhood morality, Spacing between births, Female's age at marriage time, Infants etc.

In the recent past it is seen that various researchers have been made in the study of various physical problems through mathematical modeling. Some eminent scholars are engaged in developing various Mathematical models related to problems of everyday life motivated by these works, in the present paper, we have developed a stochastic model related to human population growth.

In our society specially in India, it is seen that great emphasis is put on the hope of a male child in a family, especially if the first child is a baby female or if there are only females in the family. In this particular area; Cormack ${ }^{[5]}$ has contributed a lot. One reason for having one or more male child is the support upon which elderly can depend in the old age especially. For this particular case we have gone through the work of May ${ }^{[2]} \&$ Heer $^{[3]}$ and Mode ${ }^{[1]}$, Keyfitz ${ }^{[7]}$ Parlenge $^{[4]}$, Rick son ${ }^{[8]}$, Sheldon ${ }^{[9]}$, Guayule ${ }^{[6]}$, Krishnamurthy ${ }^{[10]}$, Zafar et al. ${ }^{[11]}$. 
The desire for male child in societies has strong bearing on family growth. For an example, if a couple has two female Childs in their family and if still decide to continue conception and child birth up to having at least one male child in the family, it leads to the family growth. In this paper, a stochastic model of intrinsic rate of population growth is developed and studied when the couples at the family level decide whether to continue child bearing or not is made on the basis of desired number of male Childs.

\section{Objective of the Model}

The main objective of the model is to control the population, which is focused on the relationship between age at marriage and intrinsic growth rate of populations where couples would stop childbearing only after having male child. It is quite encouraging from the analysis of the formulated model, that the long run growth rate of population can be scaled down drastically by increasing the mean age at marriage from 15 years to 25 years, or so. This intrinsic growth rate goes down well below one percent when females entered into wedlock at 25 years on average and maintaining a gap of 5 years on an average between births.

From the point of view of population welfare management programmers aiming to curtail the unwilling growth of population, it is important to note that even when couples in a population go for child bearing until they have one living male child, the long run growth rate of population can be monitored and scaled down considerably by firmly advocating late marriage preferably in mid -twenties and practice of enough spacing between births accompanied with an overall improvement in the level of mortality.

\section{Formulation of Stochastic Model}

The mathematical model under consideration incorporates variation in female's age at marriage, survival chance of mothers, spacing between births and early childhood morality.

The formulation of the model under consideration is done in such a way that it incorporates variation in age at marriage and family decision process based on desired number of living male Childs.

Let $w$ be a chance variable denoting life span of a woman and let us define the distribution function of $w$ by:

$$
P(W \leq w)=\left\{\begin{array}{cl}
H(w) & , 0<w<\infty \\
0 & , w \leq 0
\end{array}\right.
$$

Let variate $X(w)$ denotes the total number of live births experienced by a woman during the time interval $[0, w]$ and let $K(w)$ be its mean function defined as:

$$
K(w)=E[X(w)]
$$

For taking $X(w)$ as a stochastic process, let $\left\{W_{n}\right\}, n=0,1,2,3 \ldots \ldots$. be a sequence of variates which indicates the time at which $n^{\text {th }}$ child is born to a married woman.

Let, (i) $W_{0}=0$ denotes the point of marriage,

(ii) The sequence of variates $X_{n}=w_{n}-w_{n-1}, n=1,2,3 \ldots \ldots$. .represents waiting time between births.

We further assume that the sequence of variates $\left\{X_{n}\right\}, \mathrm{n}>1$ are independently and identically distributed with a common distributed with a common distribution function which satisfies, 


$$
F(w)=0, w \geq 0
$$

Let $A_{u}$ denotes the event that the variate $X(w)$ (the birth process for a population desiring $\times$ alive sons) stops reproduction with $u$ children and $P_{u}(x)$ be the probability of this event. Further, let $F_{u}(w)$ be the u-fold convolution of $F(w)=F_{1}(w)$ with itself then,

$$
\begin{aligned}
& P[X(w)]=i\left[A_{u}\right] f(x)=\left\{\begin{array}{cl}
F_{i}(w)-F_{i+1}(w) & , i=0,1,2, \ldots . u-1 \\
F_{i}(w) & , i=u
\end{array} \text { and } E[X(w)]=i\left[A_{u}\right]\right. \\
& E\left[X(w) A_{u}\right]=\sum_{i=0}^{u} i P[X(w)]=i\left[A_{u}\right]
\end{aligned}
$$

$$
\begin{aligned}
& \text { So, } K(w)=E[X(w)]=\sum_{u=1}^{\infty} P_{u}(x) \sum_{i=0}^{u} i P[X(w)]=i\left[A_{u}\right] \\
& =\sum Q_{u}(.) F_{u}(w)
\end{aligned}
$$

Where, $Q_{u}()=.P_{u}()+.P_{u+1}()+.\ldots \ldots . ., u \geq 1$

For incorporating variation in age at marriage and to make the stochastic process $X(w)$ to start from age at marriage described by the distribution function $I(w)$ the mean function $\mu(w)$ of this extended process $X(w)$ is given by,

$$
\mu(w)=\int_{0}^{w}(w-y) I d y
$$

The birth rate function $b(w)$ associated with $\mu(w)$ is defined by the relation,

$$
\mu(w)=\int_{0}^{w} b q d q
$$

The intrinsic population growth rate $u$ which we planned to study as a function of desired number of alive sons decided at the family level is defined as the largest real root of the characteristic equation

$$
\int_{0}^{w} e^{-u w}[1-H(w)] \mu d w=1
$$

The equation relates the population growth rate $u$, the survival function $H(w)$ and parameter of human reproduction associated with $\mu(x)$ the evaluation of $u$ from equation (8) as a function of desired number of alive sons and parameters of human reproduction is made under the following assumptions:

(i) The waiting time to marriage from birth follows an exponential distribution with probability density function. 


$$
f_{0}(y)=m e^{-m y} m>0, y>0
$$

(ii) A women is pregnant and fecund at the time of marriage,

(iii) Each conception ends in a live birth,

(iv) Waiting times to conceptions follows a negative exponential distribution with parameter $\lambda>$ 0 . There is no heterogeneity in the desired number of living male Childs.

Let, $I(q), F(q), H(q), K(q)$ and $\mu(q)$ be the Laplace-Stietljge's transforms of $I(),. F(),. H(),. K($.$) and$ $\mu$ (.) respectively, then on the basis of above assumption,

$$
\begin{aligned}
& I(q)=\frac{m}{m+q} \\
& F(q)=\frac{\lambda}{\lambda+q} \\
& {[F(q)]^{n}=\int_{0}^{\infty} e^{-q t_{0}} F d w} \\
& \mu(q)=I_{0}(q) K(q) \\
& g(z)=p_{0}+p_{1} z+p_{2} z^{2}+p_{3} z^{3}+\ldots \ldots . . \\
& h(z)=q_{1} z+q_{2} z^{2}+q_{3} z^{3}+\ldots \ldots \ldots .
\end{aligned}
$$

be the probability generating functions of $P($.$) and Q($.$) respectively. Now h(z)$ can be expressed in terms of $g(z)$ as: $h(z)=\left(P_{0}+P_{1}+P_{2}+P_{3}+\ldots \ldots\right) z+\left(P_{0}+P_{1}+P_{2}+P_{3}+\ldots \ldots\right) z^{2}+\ldots \ldots$.

$$
\begin{aligned}
& =p_{1} z+p_{2}\left(z+z^{2}\right)+p_{3}\left(z+z^{2}+z^{3}\right)+\ldots \ldots . \\
& =\frac{z}{1-z}\left[p_{1}+p_{2}+p_{3}+\ldots \ldots . .-\left(p_{1} z+p_{2} z^{2}+p_{3} z^{2}+\ldots \ldots .\right)\right] \\
& =\frac{z}{1-z}[1-g(z)]
\end{aligned}
$$

With the help of equation (11) in equation (4) we get $k(q)$ as the Laplace transform of $k(w)$ as,

$$
\begin{aligned}
K(q) & =Q_{1}\{F(q)\}+Q_{1}\{F(q)\}^{2}+Q_{1}\{F(q)\}^{3}+\ldots \ldots \ldots \\
& =h[F(q)] \\
& =\frac{F(q)}{1-F(q)}[1-g\{F(q)\}]
\end{aligned}
$$


The probability distribution $p_{u}, u=0,1,2 \ldots \ldots$ of the number of children born to couples when they continue child bearing till one male child lives, the first two years of life is found to be well described by the modified geometric distribution.

$$
p_{0}(1)=\beta \text { and } p_{u}(1)=(1-\beta)(1-\alpha) \alpha^{u-1} \quad u=1,2,3, \ldots \ldots \ldots \ldots
$$

Where $(1-\alpha)$ i.e. probability that couples has a male child which lives first two years of life and $\beta$ is the proportion of couples who never had a male child living the first two years of life. In this formulation, it is assumed that couples wait for at least two years for making decision on continuous or discontinuous of child bearing based on living of a male child for the first two years of life.

The probability generating function of this modified geometric distribution is,

$$
g(z)=\frac{\beta+(1-\beta)(1-\alpha) z}{1-\alpha z},|z| \leq \frac{1}{\alpha}
$$

From equation (10), (16), (18), we get,

$$
\begin{aligned}
K(q)= & \frac{F(q)}{1-F(q)}[1-g\{F(q)\}] \\
g(z)= & \frac{\beta+(1-\beta)(1-\alpha) z}{(1-\alpha z)} \quad \text { on puting } Z=F(q) \text { we get } \\
g F(q) & =\frac{\beta+(1-\beta)(1-\alpha) F(q)}{1-\alpha F(q)} \\
& =\frac{\beta+(1-\beta)(1-\alpha) \frac{\lambda}{\lambda+q}}{\lambda} \quad \because F(q)=\frac{\lambda}{\lambda+q} \\
= & \frac{\lambda \beta+q \beta+\lambda-\lambda \alpha-\lambda \beta+\lambda \alpha \beta}{q+(1-\alpha) \lambda} \\
g F(q) & =\frac{q \beta+\lambda-\lambda \alpha+\lambda \alpha \beta}{q+(1-\alpha) \lambda} \\
1-g F(q) & =1-\frac{q \beta+\lambda-\lambda \alpha+\lambda \alpha \beta}{q+(1-\alpha) \lambda} \\
= & \frac{q+\lambda-\alpha \lambda-\lambda-q \beta-\lambda \alpha \beta+\lambda \alpha}{q+(1-\alpha) \lambda} \\
= & \frac{q-q \beta-\lambda \alpha \beta}{q+(1-\alpha) \lambda}
\end{aligned}
$$


Hence, $K(q)=\frac{F(q)}{F(q)}[1-g\{F(q)\}]$

$$
\left[\frac{q-q \beta-\lambda \alpha \beta}{+(-\alpha) \lambda}\right.
$$

$$
\begin{gathered}
\frac{\lambda}{q} \frac{q-q \beta-\lambda \alpha \beta}{q+(-\alpha) \lambda} \\
\frac{\frac{\lambda}{q}(1-)-\frac{\lambda \alpha \beta}{q}}{+(-\alpha) \lambda}
\end{gathered}
$$

$K(q) \frac{\lambda(1 \quad \beta)}{+(-\alpha) \lambda}$

Now from equation (9), (12), (19) we have,

$$
\begin{aligned}
\mu(q) & =\frac{m}{m+q} \times \frac{\lambda(1-\beta)}{q+(1-\alpha) \lambda}=I(q) \times K(q) \\
& =A(1-\beta) m\left[\frac{1}{q+(1-\alpha) \lambda}-\frac{1}{q+m}\right] \quad \text { where } A=[m-(1-\alpha) \lambda]^{-1}
\end{aligned}
$$

The inverse Laplace transform of equation (20) gives the birth rate function,

$$
b_{1}(w)=A(1-\beta) m \lambda\left[e^{-(1-\alpha) \lambda w}-e^{-m w}\right]
$$

Which is the birth rate function when couples continue child bearing till a male child lives the first two years of life.

The probability generating function of the probability distribution of the number of children born to couples when they continue child bearing till two male child lives the first two years of life can be taken as the product of probability generating functions of independent geometric distribution given in equation (17) and it is $g(z)$ by the probability generating function. So, we get,

$$
K(q)=\frac{\lambda\left(1-\beta^{2}\right)}{q+(1-\alpha) \lambda}+(1-\beta)^{2}(1-\alpha) \times \frac{\lambda^{2}}{\{q+(1-\alpha) \lambda\}^{2}}
$$


As a consequence we get,

$$
\mu(q)=\frac{m \lambda\left(1-\beta^{2}\right)}{(q+m)+\{q+(1-\alpha) \lambda\}}+\frac{m \lambda^{2}(1-\alpha)(1-\beta)^{2}}{(q+m)\{q+(1-\alpha) \lambda\}^{2}}
$$

The birth rate function $b_{2}(w)$ when couples continue childbearing till two sons lives the first two years of life, is given by the inverse Laplace Transform of equation (23) as,

$$
b_{2}(w)=\left(v_{2}-v_{1}\right) e^{-m w}-\left(v_{2}-v_{3}\right) e^{-(1-\alpha) \lambda w}
$$

Where,

$$
\begin{gathered}
v_{1}=\frac{m \lambda\left(1-\beta^{2}\right)}{m-(1-\alpha) \lambda} \\
v_{2}=\frac{m \lambda^{2}(1-\beta)^{2}(1-\alpha)}{\{m-(1-\alpha) \lambda\}^{2}} \\
v_{3}=\frac{m \lambda^{2}(1-\beta)^{2}(1-\alpha)}{\{m-(1-\alpha) \lambda\}}
\end{gathered}
$$

Now using the result of equation (21), in equation (8), the intrinsic growth rate associated with the decision of couples to continue childbearing till a male child lives the first two years of life is the largest real root of the equation:

$$
\begin{aligned}
& \int_{0}^{\infty} e^{-u_{1} w}\{1-H(w)\} b_{1}(w) d w=1 \\
& \text { or } \\
& A(1-\beta) \lambda m \int_{0}^{\infty} e^{-u_{1} w}\left\{e^{-(1-\alpha) \lambda w}-e^{-m w}\right\}\{1-H(w)\} d t=1
\end{aligned}
$$

or

$$
u_{1}^{2}+B u_{1}+C=0, \text { where } \quad B=2 l+m+(1-\alpha) \lambda, C=\{l+(1-\alpha) \lambda\}(l+m)-(l-\beta) m,
$$

By assuming that life span distribution follows negative exponential distribution with mean life $1 / l$.

Similarly the result of equation (24) in equation (8), the intrinsic growth rate $u_{2}$ associated with the decision to continue childbearing till two male child lives the first two years of life is the largest real root of the equation, 
$\int_{0}^{\infty} e^{-u_{2} w}\{1-H(w)\} b_{2}(w) d w=1$

or

$u_{2}^{3}+\left[l+m+2\{l+(1-\alpha) \lambda\}-v_{3}\left(v_{1}^{\prime}-v_{3}^{\prime}\right)\right] v_{2}^{2}+2(l+m)\{l+m(1-\alpha) \lambda\}+\{l+(1-\alpha) \lambda\}^{2}$

$-\left(v_{1}^{\prime}-v_{2}^{\prime}+v_{3}^{\prime}\right) u_{2}+(l+m)\{l+(1-\alpha) \lambda\}^{2}-u_{3}(2+m)-\left(v_{1}^{\prime}-v_{2}^{\prime}\right)\{l+(1-\alpha) \lambda\}=0$

or

$u_{2}^{3}+D u_{2}^{3}+E u_{2}+F=0$

where

$$
\begin{aligned}
& v_{1}^{\prime}=m(1-\beta), v_{2}^{\prime}=\frac{m \lambda^{2}(1-\beta)^{2}(1-\alpha)}{m-(1-\alpha) \lambda} \\
& D=\left[l+m+2\{l+(1-\alpha) \lambda\}-v_{3}\left(v_{1}^{\prime}-v_{3}^{\prime}\right)\right] \\
& E=2(l+m)\{l+m(1-\alpha) \lambda\}+\{l+(1-\alpha) \lambda\}^{2}-\left(v_{1}^{\prime}-v_{2}^{\prime}+v_{3}^{\prime}\right) \\
& F=(l+m)\{l+(1-\alpha) \lambda\}^{2}-u_{3}(2+m)-\left(v_{1}^{\prime}-v_{2}^{\prime}\right)\{l+(1-\alpha) \lambda\}
\end{aligned}
$$

\section{DISCUSSION OF THE MODEL}

The model proposed and formulated in the preceding section illustrates the decision made at the family level about having at least one male child and side by side the spacing between births \& the effect on population growth. It also describes critical factors which need to be monitored at the optimum level to curtail the alarming growth of population. The factors included in the decision are social factors which are listed below:

(i) Age at marriage time

(ii) Number of male Childs

(iii) Spacing between births,

(iv) Survival of mother (During child bearing period).

This is taken as the average years of life expected to live after 15 years.

The mean age at marriage of females and spacing between births are however allowed to vary with the intention of identifying the right combinations and parameters of human reproduction process to arrive at an optimum rate of population growth for various combinations of biosocial parameters of models, which are consistent with the prevailing figures in India, intrinsic rate of population growth are generated through the proposed models. Now a day's intrinsic rate of population growth is very high as couples go for having children until they have one male child in the family, which can be seen in many ways. First let us examine the relationship between chance of a child dying in the first two years of life and intrinsic growth rate. At this step, it is interesting to observe that there exists direct relationship between 
the level of child mortality and intrinsic growth rate. In the course of the study, we confirmed the popular hypothesis that in populations where the level of child mortality is high, couples go for larger number of children to ensure a live of at least one male child in the family, thereby increasing intrinsic rate of growth of such populations. As for as the effect of births spacing on intrinsic growth rate is concerned it is evident that the later decreases with the increase in spacing between births.

\section{CONCLUSION}

It is encouraging particularly to observed that the proposed model explains fairly well the sensitiveness of the bio-social parameters included in the formulation. However, it is also observed that there is a marked difference in the values of intrinsic growth rate when couples go for at least two male Childs as compared to corresponding figures of one male child, even more than double of the corresponding figures for one male child. On the basis of the proposed model the following conclusions are drawn:

(i) The intrinsic growth rate varies inversely with the mean age at marriage, as a result long run population growth can be curtail by increasing age at entering wedlock preferably after twenty years for the female.

(ii) The intrinsic growth also varies inversely with births spacing, consequently with births spacing consequently the growth of population can be scaled down considerably by advocating firmly longer spacing between births in the range of 3 to 5 years.

(iii) The more the chance of living of infants, the lower is the growth rate of population consequently, population growth can also be retarded by ensuring survival of newborn babies.

\section{REFERENCES}

1. Mode, C.J. 1974. A Study of a Malthusian Parameter in Relation to Some Stochastic Models of Human Reproduction. Theoretical Population Biology, 3: 300-325.

2. May, D.A. and Heer, M. 1968. Son Survivorship and family size in India: A Computer simulation. Population Studies, 22: 199-210.

3. Heer, D.M. and Smith, D.O. 1968. Mortality Level, Desired family size and population Increase, Demography, 5: 104-121.

4. Parlenge, J.Y. 1985. Mortality Levels and Fertility Goals, Demography, 30: 625-635.

5. Cormack, M. 1982. The Hindu Women Bombay.

6. Guilyole, M.J. 1990. Population growth rate and Mortality levels, 30: 425-435.

7. Keyfitz, N. 1977. Applied Mathematical Demography, John Wiley and Sons, New York.

8. $\quad$ Rickson, R.E. 1984. A model on Intrinsic growth rate, 20: 525-536.

9. Sheldon, R.N. 1994. Stochastic Process; Second edition Wiley series in probability and Mathematical Statistics.

10. Krishnamoorthy, S. 1979. Mortality Level, Desires for Surviving Son and Rate of Population Increase. Population Studies, 35: 652-671.

11. Zafar, A. 2004. Differential equations and their applications, $2^{\text {nd }}$ ed. Prentice Hall, New Delhi. 
